\title{
NEW PUBLICA'TIONS.
}

\section{HIGHER MA'THEYATICS.}

Bökns (C.). Die Ordnungscurven des Nullsystems, ihre Vertheilung im Ritume und ihre Construction. Frunkenthal, 1892. 8vo. $26 \mathrm{pp} .111$.

Mk. 1.20

Bongmeyer (J.). Geometrische Untersuchung über den Ort der Fusspunkte der Lote, welche von einem Punkte auf die Strahlen eincr linearm Congruenz gefält werden. Hildesheim, 1893. 8vo. 51 pp.

Mk. 1.50

Cauchy (A.). Euvres complètes. 1re série. Vols. VIII and IX: Extraits des Comptes rendus de l'Académie des Sciences. Paris, Gauthier-Villars, 1893. 41\%. Each volume Fr. 25.00

2e série. Vol. X.: Résumés analytiques de Turin. Nouveaux exercices de Prague (Alémoire sur la dispersion de la lumière). Paris, Gauthier-Villars, 1893. 4to.

Gérard (L.). Sur la géométrie non-Euclidienne. Paris, 1892. 4to $115 \mathrm{pp}$. III.

Mk. 4.50

Gourzmer (A.). Veber gewisse partielle Differentiagleichungen höherer Ordnung. Halle, 1893. 4to. 22 pl. Mk. 1.20

Jondan (C.). Cours d'analyse de l'École polytechnique. (In 3 vols.) Vol. I. Calcul différentiel. 2e édition, entièrment refondue. Paris. Gatuthier-Villars, 1893 . 8vo. 18 and 612 pp. Ill. Fr. 17.00

Kraft (F.). Abriss des geometrischen Kalküls. Nach den Werken Hermann Grassmanns bearbeitet. Teipziq. Teubner, 1893. 8vo. 12 and $255 \mathrm{pl}$. Ill.

Mk. 6.00

I Acindan (R.). An elementary trenlise on modern pure geometry. New York, Macmillan, 1893. 12mo. 10 and $288 \mathrm{pp.} \quad \$ 2.25$

Lucas (E.). Récréations mathématiques. Vol, III. Paris, GauthierVillars, 1893. 8vo.

Fr. 6.50

Martone (M.). Introduzione alla teoria delle serie. Parte II. Catanzaro, 1892.

Fr. 5.00

Mrnkowskı (II.). Geometrie der Zahlen. Eine Festschrift zum siebzigsten Gebnrtstage von Herm Charles Hermite. Leipzin, Teubner, 1893. . 8 vo.

Schemdenbers (C.). Neue Behandlung der hypergeometrischen Function auf Grund ihrer Definition durch das bestimmle Integral. Göttingen, 1892. 8vo. $68 \mathrm{pp}$.

Wulink (A.). The world of the unseen. An essay on the relation of higher space to things etermal. New York, Macmillan, 1893. 12mo. $184 \mathrm{pl}$.

$\$ 1.25$

\section{ELEMENTARY MATHEMATICS.}

Beuikov (A.) and Nathing (A.). Text-book of algebra, with a collection of exercises. [Russian.] St. Petersburg, 1893. 8vo. 6 and $402 \mathrm{pp}$. 
C'LAkK (A. D.). Army, Woolwich, and Civil Service readers: geometrical problems selected from the examination papers of the last twenty years. New York, Longmans, 189:3. Sro. 63:3 pp. (!1.

GRÉry (A.). Compositions domnées depuis 1872 anx eximnens de saint Cyr. Alec̀bre et géométrie. (Énoncés et solutions.) 21 ed. Paris, Gauthier-Villars, 1893. Sro. Ill. Fr. 2.jt

IAla (W. S.). Mensuratiou. Boston, Ginn, 189\%. 6 and 6\% pp. 12mo. (Mathematical series.) C].

$\$ 0.5 \mathrm{i}$

Irata (L.). Del circolo edelle sue proprietit trigonometriche. Roma. Perino, 1892. 8vo. $16 \mathrm{pp}$. 3 plates.

Lasan't (C. A.). Recueil de problèmes de mathématiques. chassies par. divisions scientitiques. Vol. I. Arithmétique, algèbre élémen. taire, trigonométrie. Paris, 1893. Svo. Fr. 2.50

Lisoviralide (G.). Grundzüge der 'Trigonometrie und stereometrie. Halle, 1893. 8vo. Ill.

MK. 1.20

Mrchetsex (P.). Die bestimmten algebraischen Gleichungen des 1sten bis 4ten Grades, Nebst Anhanx: Lnbestimmte Gleichun gen. Hammover, 1892. 8vo. 8 and $306 \mathrm{pp}$. Mlk. 4.00)

Mraxe (IV.J.). Elements of arithmetic for primary and intermediate classes in public and private schools. New Tork, American lsook Company [1893]. 12mo. $240 \mathrm{pl}$. (1.

Xeumax (K. W.). Lehrbuch der allgemeinen Arithmetik und Alge bra. 6th ed. Bremen, Heinsius, 1892. $215 \mathrm{pl}$. $1 \mathrm{k} .2 .84$

Peterseñ (J.). Analytisk Plangeometri. 3 d ed. Part 1. Kiöben. havn, 1893. Svo. 96 pp. Mk. 2.50

Pincherie (S.). Algebra complementare. Parte I : Analisi algebrica. Milano, Hocpli, $1893.16 \mathrm{mo} .8$ and $182 \mathrm{pl}$. Ill. $1 \mathrm{lk} .1 .50$

REmbaczA (M.). Krétko zebrana historya gocometryi wykresinéj. [Brief history of descriptive geometry.] Part II. Simishan, 1891. Svo. $64 \mathrm{pl}$.

Ilk. 2.00

Russel. (J. W.). An elementary treatise on pure geometry, with numerous examples. London, Frowde, 1893. 8vo. 3338 pp.

$10 s .6 \dot{\alpha}$.

Winss (H.). Iandbook of algebra. London, 189:. Svo. 26s fpp. Cl.

is. 6 et.

\section{APPLIED MATHENA'TICS.}

Apper, (P.). 'Traité de mécanique rationnelle. (In 3 vols.) Vol. I : Statique. Dynamique du point. Paris, Gauthier-Villars, 1893. 1er fasc., $320 \mathrm{pp}$.

Fr. 14.00

BARlow (C. W. C.) and BRYAN (G. H.). Elementary mathematical astronomy. With examples and examination papers. London. Clive, 1893 [Univ. Corr. Coll. Tutorial Ser.]. 8vo. 446 pp. 8s.6a.

Bauschinger (J.). Untersuchungen über den periodischen Kometen $1889 \mathrm{~V}$ (Brooks). Part I : Definitive Bahnbestimmungen der Hauptkometen aus den Erscheinungen 1889 bis 1891 . Miünchen. Franz. 4to.

Mk. 5.00 
Brugaer (C.). Die Erhaltung der Energie, das Grundprincip der nenen Naturlehre. Einsiedeln, 1893. 4to. 36 pp. Mk. 2.20

Dexarçay (J.). Théorie mathématique des guillotines el obturateurs centraux droits. Paris, 1892. 8 vo. 4 and 59 pp. Ill. Mk. 1.80

Indra (A.). Neue ballistische Theorien. Beiträge zum Studium newer Probleme der immeren und atusseren Ballistik. Theil I : Analytische Theorie der Wärmeleitung in Geschützrohren. Pola, 1893. Svo. 14 and $17 \%$ pp.

Mk. 6.00

Necranas (.). Beitrige zu einzelnen Theilen der mathematischen Physik, insbesondere zur Elektrodynamik und Hydrodynamik, Elektrostatik und magnetischen Induction. Leipzig, 'Teubuer, 1893. 8vo. 8 and 314 pp. Ill. Mk. 10.00

Rasmussen (A. H. M.). Lären om Skibsdampmaskinen. 2te omarbejdede udgave. Ulgiven ved Marineministeriets foranstaltning. Kjöbenhavn, 1893. 8vo. 588 pp. 33 plates.

RUdolpu (H.). Anwendung der akustischen Methode der Längenmessung zur Bestimumung der linearen Ausdehnungscoefficienten von Stäben unter Benutzung einer einfachen Vorrichtung zur Ermittelung der Acnderung der Schwingungszahl einer Saite. Marburg. 189. 8vo. 49 pp. 1 plate.

Skerig (E.). Nolekularkrifte. Physikalisch-chemische Studie der verschiedenen Körperzustände. 2d ed. Berlin, Friedlinder, 1893.

Mk. 2.40

Förken (H.). Dic Centralbewegung. Entwicklung der einschlägigen Gesetze und Zusammenstellumg der auf ihmen beruhenden Versuche und Erscheinungen. Berlin, 1893 . 4to. 36 pp. 2 plates. Mk. 1.50

WAiso (F.). Modern meteorology : an outline of the growth and present condition of some of its phases. New York, Scribner, 1893. 8vo. 21 and $460 \mathrm{pp}$. (Contemporary science series.) Cl.

Werber (W.). Werke. Herausgegeben von der Königl. Gesellschaft der Wissenschaften zu, Göttingen. (In 6 vols.). Vol. III.: Galvanismus und Elektrodyuamik. Theil 1 , besorgt durch $\mathrm{H}$. Weber. Berlin, Reimer, 1893 . 8vo. 12 and 676 pp. 1 plate. Mk. 20.00

Wernicke (A.). Beilräge zur Theorie der centrodynamischen Kör. per. Braunschweig, Vieweg, 1892. 4to. 36 pp.

Winkemann (A.). Handbuch der Physik. Herausgegeben unter Mitwirkung von F. Auerbach, F. Braun, S. Czapski, K. Exuer, n. A. (In 3 vols.) Vol. III. : Elektrizität und Magnetismus. Part 1. Breslau, Trewendt, 1893. 8vo. 546 pp. Ill. Mk. 15.00

Zunger (C. V.). Le système du monde electrodynamique. Prague, 1893. 4 to. 24 pp. 1 plate. 